\title{
11 \\ Real-Time Estimation of the Link Capacity in Multimedia Networks
}

\author{
Piergiulio Maryni \\ DIST-University of Genoa \\ Genoa, Italy, tel.3910 3532732, e-mail: pg@dist.unige.it \\ Giovanni Pacifici \\ IBM - Watson Research Center \\ New York, USA, tel.19147847202, e-mail: giovanni@watson.ibm.com
}

\begin{abstract}
In order to guarantee quality of service, we must be able to calculate the link capacity, i.e., the total number of calls of different types that can be admitted on a single link at any given time. In this paper we use the schedulable region to represent the link capacity, and, we present a new approach for computing the schedulable region. Our methodology relies on real-time quality of service measurements to dynamically compute the size and shape of the schedulable region. We do not make any assumptions on traffic source models or scheduler operations.
\end{abstract}

\section{Keywords}

Quality of Service estimation, Multimedia Networks

\section{INTRODUCTION}

Guaranteeing Quality of Service (QoS) is the main challenge in future broadband networks. When several calls are statistically multiplexed into a single channel, there is the need to find out if and how the telecommunication system can guarantee their QoS constraints. In other words the problem is how many calls of a given type can be admitted to the link at any given time. In this paper we focus on the so-called "low-level" QoS and in particular on the cell flow level of an ATM network. At this level, the quantities of interest are the probability of exceeding a given value of delay and the probability of having cells blocked due to the lack of transmission resources.

Each node of a telecommunication network has a finite switching capacity, due to the finite processing power and the finite link capacity. This switching capacity strongly depends on traffic statistics and QoS constraints. In a circuit 
switching, this maximum switching capacity is easy to find, while in a packet switched framework in which a set of QoS have to be guaranteed, the switching capacity is, in general, unknown and the problem is to find it.

The problem of finding the link capacity has been largely considered and many examples can be found in the literature (see for example (D. Ferrari et al. 1990, R. Guerin et al. 1991)). All these techniques rely on very strong assumptions on the system structure (e.g., markovian behavior of input flow, FIFO scheduling, simple server model), and they generally need a computational effort that might lead to a non effective utilization in a real-time framework. A common problem with these techniques is that the analysis generally focuses only on the entry points of the network since source models are usually non effective as the flow goes deeply into the network. This is mainly due to the fact that each scheduler might change the flow properties that cells had when they entered the network (J.F. Kurose 1993). Several techniques have been proposed to overcome this problem (see for example (A. Parekh et al. 1992)). Other techniques keep track of the variations that the single flows have as they travel along the network (J.F. Kurose 1992). They do not try to preserve the characteristics of the flow, but instead they are able to compute bounds on a node-by-node fashion.

The approach which we are considering in this paper is to find the link capacity of a node without relying on any mathematical model of sources or on the presence of a given scheduler, but only by means of measurements on the QoS. It is worth to point out that by means of measurements it is not possible, in general, to find the real switch capacity but only an estimation of it. On the other hand, this approach, has the notable advantage of being applicable to a wide range of situations, since it does not require any particular constraint on the switch components such as the scheduling policy, the buffer size or the buffer management policy. In (J. M. Hyman et al. 1991), given a set of traffic classes, the switch capacity is estimated by means of a simulation process and described by a data-structure called "schedulable region". The main problem with this approach is that, if the traffic statistics of a given class change, the switch capacity previously found is no longer reliable. Another simulation therefore would have to be run with the new traffic characteristics. This problem could be solved if we were able to estimate the link capacity in real time. In this paper we analyze this problem, suggesting a framework in which this complex estimation task can be carried out.

In Section 2 we introduce the framework and the model of the switching system we will consider. In Section 3 we describe the estimation approach we propose, while in Section 4 we present some numerical results. Section 5 draws the conclusions of this work. 


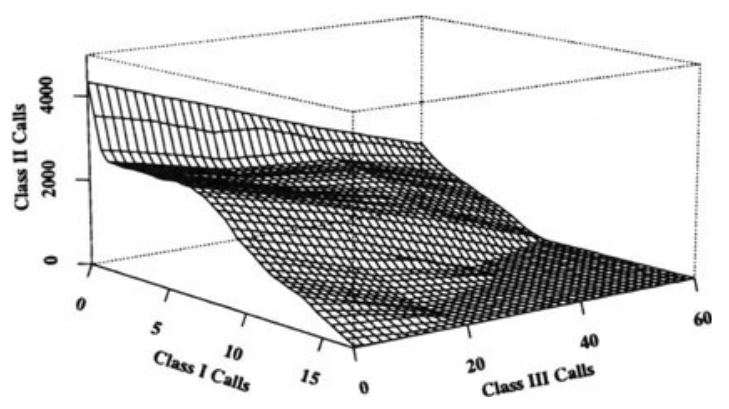

Figure 1 An example of schedulable region.

\section{A FRAMEWORK FOR LINK CAPACITY ESTIMATION}

In this paper we represent the link capacity using the schedulable region. The schedulable region of a system is the set of call configurations, for the various traffic classes, for which QoS would be guaranteed. In Figure 1 is depicted an example of schedulable region of a link over which three traffic classes are multiplexed. Over that link, if no calls of class I and III are present, than more than 4000 class II calls can be multiplexed, and all of them will have their QoS guaranteed.

The link capacity is a function of a number of parameters such as the link speed, the scheduling policy, the buffer policy, the traffic statistics and the buffer size, and therefore it changes as a result of changes in one of them. The problem we will analyze in this paper is to keep track of these variations in real time in order to maintain consistency on the network. It helps to think of a system that once in steady state is perturbed. There are no general rules that enable to compute the new boundary of the schedulable region given that some parameters have changed.

In order to keep track of the schedulable region in real time is therefore necessary to exploit estimation tasks. Estimating the schedulable region means to estimate what is the maximum load achievable by the multiplexer, and this, in turn, means to understand how QoS changes as the number of calls increases. The system therefore has to perform two estimation tasks. The first is the estimation of the current level of QoS which is being offered to the calls in progress. The second task is the estimation of the maximum number of calls that could be accepted by still guaranteeing QoS constraints. In this paper we will focus on the second estimation task.

\subsection{The system model}

The key point of the underlying switch model is the presence of a multiplexer that is designed to work with a finite set of traffic classes. The traffic class is an attribute that reflects the quality of service parameters and that it is defined 
by a set of quality of service constraints. For the sake of simplicity, we restrict our analysis to a link shared by two different classes of real-time traffic: voice and video. In such a framework, QoS are given in terms of maximum cell delay and maximum probability of exceeding such delay boundary. We will refer to the maximum delay for class $h$ as $S^{h}$ and to the maximum probability of exceeding $S^{h}$ (i.e., the QoS constraint for class $h$ ), as $\epsilon^{h}$. Hence, for class $h$, QoS will be guaranteed if:

Prob[celldelay $\left.>S^{h}\right] \leq \epsilon^{h}$

We will moreover express the load in terms of number of calls for the various traffic classes multiplexed in the system. We will refer to the number of calls for class $h$ as $K^{h}$. We will study two different scheduling disciplines: FIFO and SPS. Moreover, we will focus on delays therefore assuming infinite buffer size. The server models an ATM link which transmits cells at a constant rate of $\mu$ $\mathrm{bits} / \mathrm{sec}$. The scheleton of the model of such a multiplexing system is depicted in Fig. 2. In all the experiments we will show in the remainder of the paper, the link speed $\mu$ is equal to $100 \mathrm{Mbits} / \mathrm{sec}$. The voice source is generated with

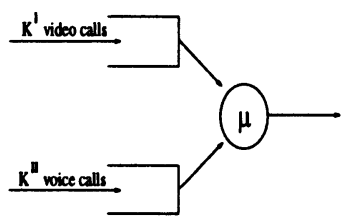

Figure 2 The model the multiplexing system.

the well-known On-Off model with exponential state transition rates. When in Off state, the source is idle, while when in On state the transmission takes place at a rate of $64 \mathrm{Kbit} / \mathrm{sec}$. The $\mathrm{On}$ and Off average periods are respectively $0.352 \mathrm{sec}$ and $0.653 \mathrm{sec}$. The generated flow is supposed to be inserted in the payload field of ATM cells. The video source generates 24 frames per second and each of them is associated a variable number of bit. The number of bit per frame is a random variable $\nu$ that comes from a given distribution and that has a correlation given by a particular extraction rule. Such video stream is generated by using the TES (Transform, Expand, Sample) technique (A. A. Lazar et al. 1994). We considered three different video sources, which in turn correspond to three different distributions for the variable $\nu$. Let us call them Video1, Video2 and Video3. Figure 3 show the three distributions for the three different video sources. In particular, Videol and Video2 have a peak rate equal to 56000 bits/frame, while Video3 has a peak rate of 64000 bits/frame. It is worth noting that for Video1, the probability of exceeding $50000 \mathrm{bits} / \mathrm{sec}$ is extremely low. As regards average rates, Video1 and Video 2 have an average rate of about 23000 bits/frame while Video3 has an average rate of about 44000 bits/frame. Notice that actual video sources might have higher peak 


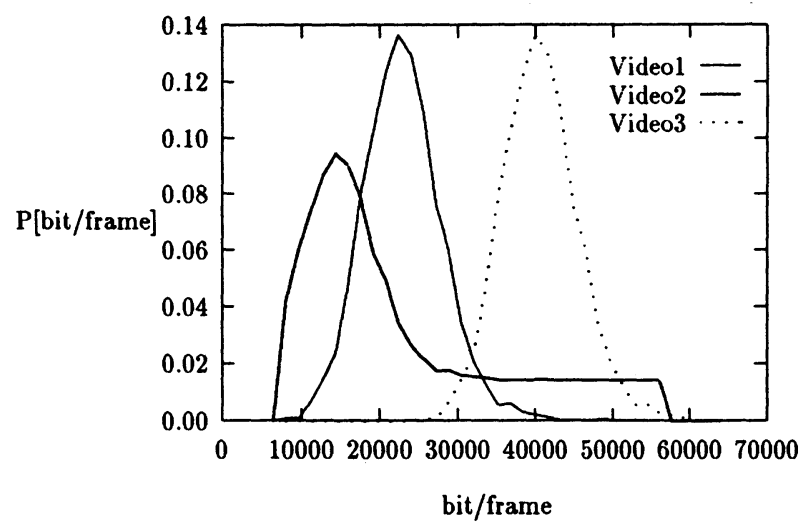

Figure 3 The distributions for the three video sources

rates. We adopted these figures in order to test in a more reliable way the effect of statistical multiplexing.

\section{A NEW APPROACH: MODELING DELAY DYNAMICS}

The approach we are considering in this work is to directly focus on delay distributions, trying to analyze their dynamics. We want to find a parametric model for delay distributions and to study how the model parameters change with variations both in the load and in traffic statistics of the input streams.

\subsection{The delay distribution model}

The model we suggest in this paper describes delay distribution for the traffic class $h$ as a collection of $\mathrm{N}$ exponential functions, each of them representing a portion of the delay axis. More in particular, if $\operatorname{pdf}^{h}(x)$ is the delay probability distribution for Class $h$, then the model can be expressed in the following way:

$\operatorname{pdf}^{h}(x)=\sum_{i=1}^{N^{h}} e^{a_{i}^{h}+b_{i}^{h} x} 1_{\left[x_{i}^{h}, x_{i+1}^{h}\right)}(x)$

Figure 4(a) gives a pictorial representation of the model expressed in equation (2), while in Figure 4(b) is shown an example of an actual delay distribution which comes from an experiment with $K^{I}=80, K^{I I}=1980$, and SPS scheduler. The shape of this delay distribution is clearly represented by the model (2). The two lines represent the exponential functions (in this case two) that approximate the delay distribution. We have observed the same behavior of the pdf for various traffic load characteristics as we will present 
later in Section 3.2. In our case, the schedulable region is defined by the set of

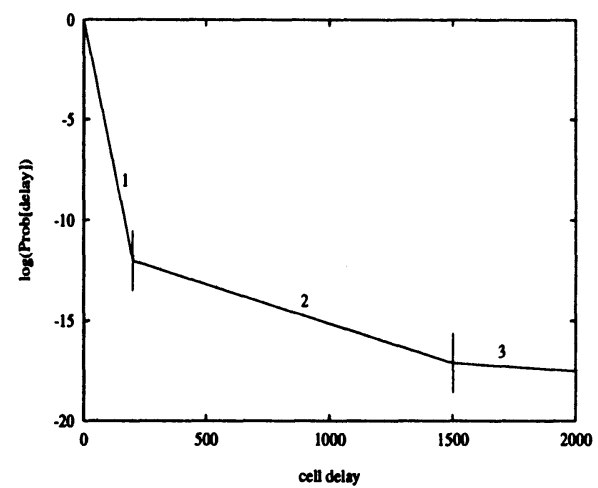

(a)

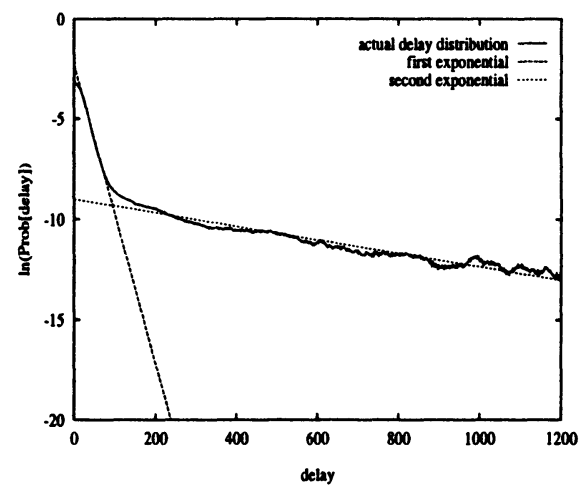

(b)

Figure 4 The model for the delay distribution. (a) Pictorial representation; (b) Example of delay distribution for Class II traffic.

$\left(K^{I}, K^{I I}\right)$ pairs such that the delay constraints for all the traffic classes are guaranteed. With respect to our delay distribution model (see equation (2)), this turns into the following:

$\int_{s^{h}}^{\infty} \sum_{i=1}^{N^{h}} e^{a_{i}^{h}+b_{i}^{h} x} 1_{\left[x_{i}^{h}, x_{i+1}^{h}\right)}(x) d x \leq \epsilon^{h}$

Equation (3) has to be satisfied for each traffic class $h(h=I, I I)$. To find the boundary of the schedulable region we first fix the value of $K^{I}$ and find the maximum value of $K^{I I}$ that satisfies (3) for $h=I$,II. We then do the same operation, fixing $K^{I I}$, to find the maximum value for $K^{I}$.

\subsection{Delay distribution dynamics}

In Section 3.1 delay distributions have been modeled as a collection of exponential functions. We have now to investigate how all these functions are affected by a change in the load. In other words, we have to find a model for the parameters of the exponential functions able to give us an insight on how they depend on the load. We have then to exploit such a model in order to estimate the boundary of the schedulable region. More specifically, notice that the parameters $a_{i}^{h}$ and $b_{i}^{h}$, in equation (3) are function of the load (i.e., $\left.K^{I}, K^{I I}\right)$. Since our goal is to predict the boundary of the schedulable region before actually reaching it, we have to determine how $a_{i}^{h}$ and $b_{i}^{h}$ change when 
the load is increased. It is worth to point out that also $x_{i}^{h}$ and $x_{i+1}^{h}$ depend on the load even if, from our experiments, we noticed that the effect of varying the load is small as regards them. Let us concentrate, for the time being, on the parameters $a_{i}^{h}$ and $b_{i}^{h}$ : in our first experiment, we load the system with the traffic classes described in Section 2.1, with SPS scheduler. Figure 5(a) shows how the pdf of Class II changes as the load increases. Fig 5(b) the approximation of equation (2) for the same experiment. Labels " 1 ", "2" and "3" both in Figures 5(a) and 5(b) refer respectively to $\left(K^{I}, K^{I I}\right)=(80,1980)$, $\left(K^{I}, K^{I I}\right)=(80,1960)$ and $\left(K^{I}, K^{I I}\right)=(80,1940)$; the scheduling policy is SPS. Let us assume, for this experiment, that the pdf, for $h=\mathrm{II}$, is composed

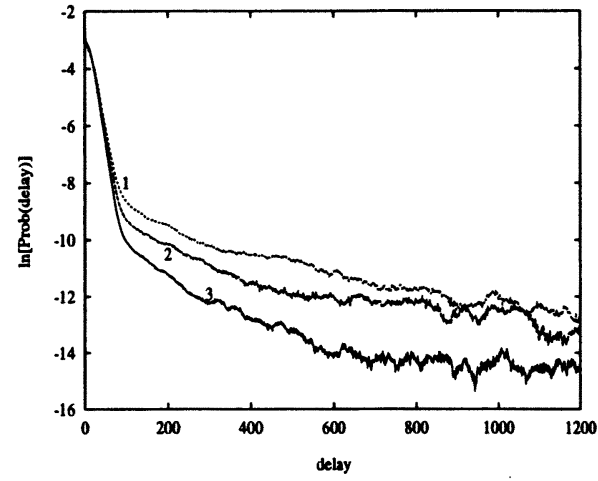

(a)

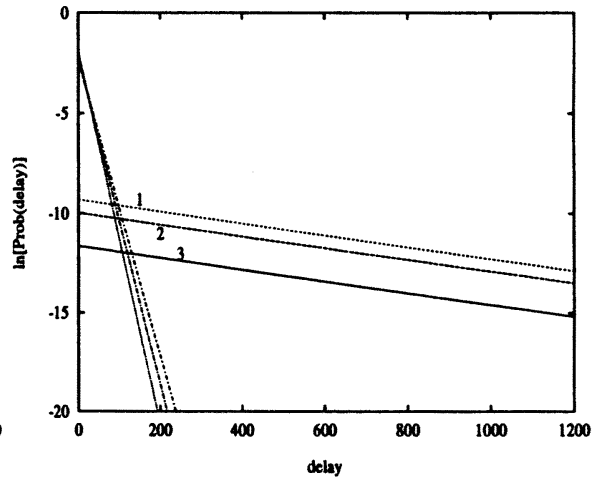

(b)

Figure 5 Delay distributions with increasing load (a) Actual distributions; (b) Model

of two exponential functions, that is:

$\operatorname{pdf}^{I I}(x)=e^{a_{1}^{I I}+b_{1}^{I I} x} 1_{\left[x_{1}^{I I}, x_{2}^{I I}\right)}(x)+e^{a_{2}^{I I}+b_{2}^{I I} x} 1_{\left[x_{2}^{I I}, x_{3}^{I I}\right)}(x)$

Figures $6(\mathrm{a}, \mathrm{b}, \mathrm{c}, \mathrm{d})$ show how the parameters $a_{1}^{I I}, b_{1}^{I I}, a_{2}^{I I}$ and $b_{2}^{I I}$ change as the load is increased. The hypothesis that we make is that, in the range of load investigated, the behavior of the model parameters is linear, with respect to the load. More specifically:

$$
\begin{aligned}
a_{i}^{h} & =\eta_{a_{i}^{h}}+\nu_{a_{i}^{h}} K^{h} \\
b_{i}^{h} & =\eta_{b_{i}^{h}}+\nu_{b_{i}^{h}} K^{h}
\end{aligned}
$$

This is an heuristic assumption that comes out from a number of experiments we carried out. The problem turns now into finding the values of $\eta_{a_{i}^{h}}$, $\nu_{a_{i}^{h}}, \eta_{b_{i}^{h}}$ and $\nu_{b_{i}^{h}}$. If we want to find them by only utilizing measurements, we 


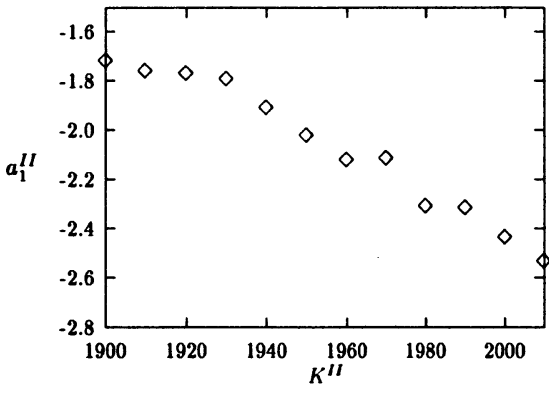

(a)

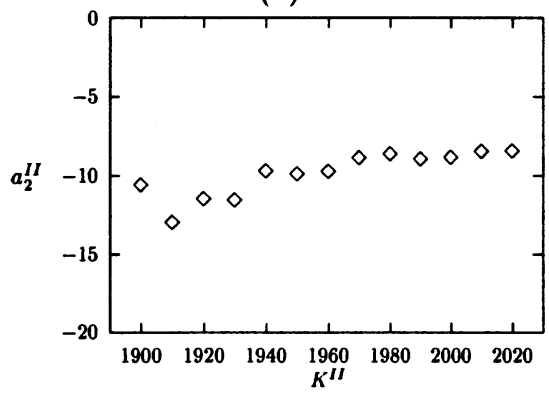

(c)

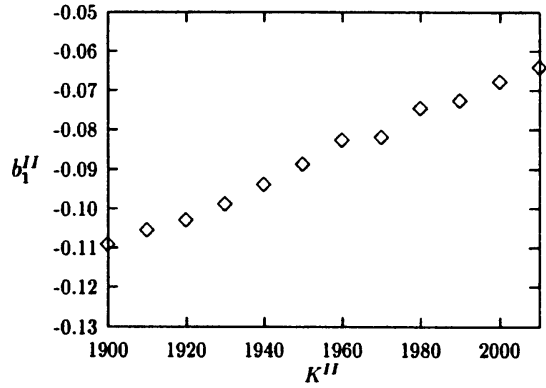

(b)

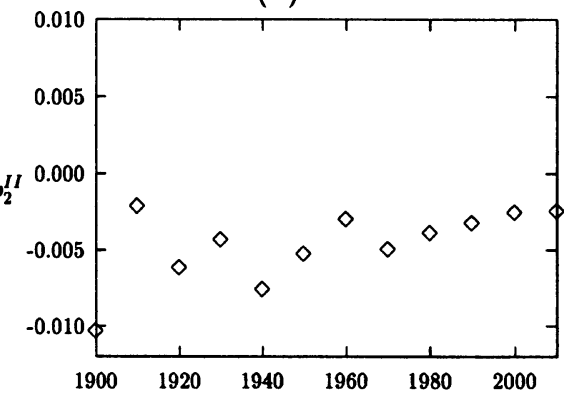

(d)

Figure 6 Parameters $a$ and $b$ as a function of the load. Scheduler=SPS, $K^{I}$ $=80, \mu=100 \mathrm{Mbits} / \mathrm{s}$. (a) $a_{1}^{I I}$; (b) $b_{1}^{I I}$; (c) $a_{2}^{I I}$; (d) $b_{2}^{I I}$;

have to perform a fitting operation among the measured values of $a_{i}^{h}$ (to find $\eta_{a_{i}^{h}}$ and $\nu_{a_{i}^{h}}$ ) and $b_{i}^{h}$ (to find $\eta_{b_{i}^{h}}$ and $\nu_{b_{i}^{h}}$ ): whenever a new load condition occurs, a new fitting task is performed and $\eta_{a_{i}^{h}}, \nu_{a_{i}^{h}}, \eta_{b^{h}}$ and $\nu_{b^{h}}$ are properly adjusted. Figure 7 shows this task for different values of the load. Notice that,

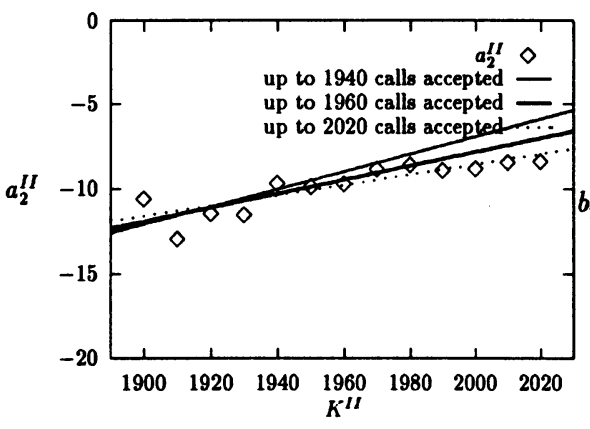

(a)

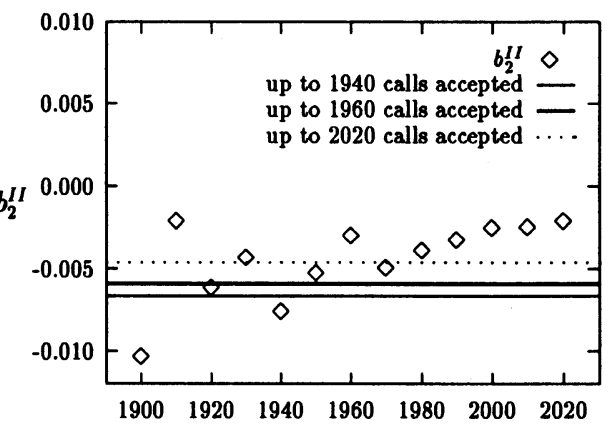

(b)

Figure 7 The model for the delay distribution. (a) Various fitting phases for $a_{2}^{I I}$; (b) Various fitting phases for $b_{2}^{I I}$.

in this example, $\nu_{b_{i}^{n}}$ has been assumed to be zero. Each time we add calls to 
the system (i.e., in this case as $K^{I I}$ increases), we can collect new information that adjust our estimation. By utilizing the various approximation obtained with different values of $K^{I I}$, and by means of both (4) and (3), we are able to estimate the schedulable region.

\section{EXPERIMENTAL RESULTS}

In this Section we report and discuss some numerical results on specific examples. More in particular we show how the estimating mechanism is able to refine its estimation as the load increases. We use the system described in Section 2.1 in which two traffic classes are statistically multiplexed over a link. For the sake of simplicity we only consider variations of one traffic class keeping the other one fixed. More specifically, we keep Class I traffic fixed to 80 calls (i.e., $K^{I}=80$ ) and we vary the number of Class II calls. Each time Class II load increases, we perform the mechanism described in Section 3 in order to update the estimation of the schedulable region. As the system acquires more information (i.e., the load increases) it refines the estimation of the boundary of the schedulable region. We expect, therefore, to obtain rough estimations when the load is low (i.e., deep inside the schedulable region) which turn into better ones as the load increases. Class I and II load is represented by video and voice calls, respectively, modeled as described in Section 2.1. The three different types of video sources described in Section 2.1 are considered. Both FIFO and Static Priority (SPS) schedulers have been taken into account. We always consider to have an infinite buffer. As regards QoS, both video and voice cells are dropped when they experience a delay greater than $2 \mathrm{~ms}$., and QoS are guaranteed when the dropping probability does not exceed 0.005 for video and 0.05 for voice (i.e., $\epsilon^{I}=0.005, \epsilon^{I I}=0.05$ ). In order to be able to test the efficiency of the estimation mechanism, we computed beforehand the "real" schedulable region with an exhaustive method and very long simulations.

Figure 8 shows the results of the estimating mechanism, when SPS scheduler is adopted (the horizontal line in the plot represents the "real" schedulable region). Class II load has been increased from a point well below the schedulable region to the point on the boundary of the schedulable region. Notice that the range of the $\mathrm{X}$ axis of the three cases is different: this is because different traffic statistics (i.e., Video1, Video2, and Video3) lead to different schedulable regions.

We observe that when Class II load is small, our approach underestimates the boundary of the schedulable region. As the Class II load increases (i.e., as the load approaches the schedulable region boundaries) the estimation succeeds in correctly predicting the boundary of the schedulable region. It is worth noticing that, as regards QoS measurements, in this experiment we only measured Class II delay: SPS scheduler, in fact, always gives priority to Class I cells which, therefore, never experience delay. This means that, in 
estimating the boundary of the schedulable region, we exploit (3) for $h=2$ only (i.e., for Class I QoS are always guaranteed).

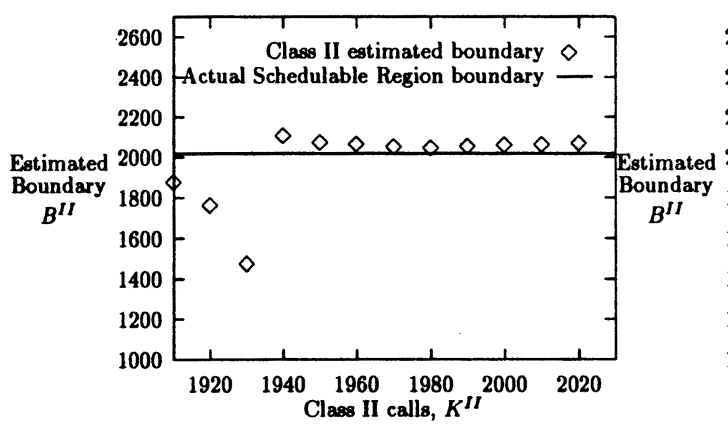

(a)

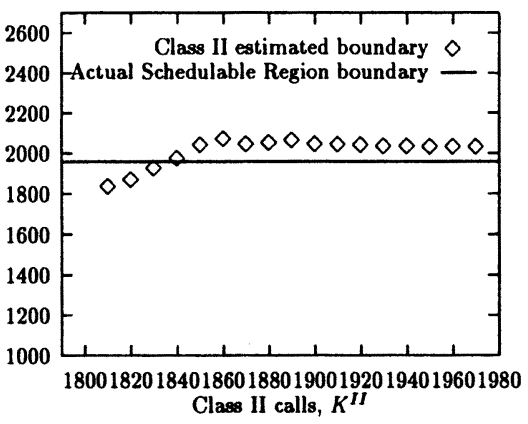

(b)

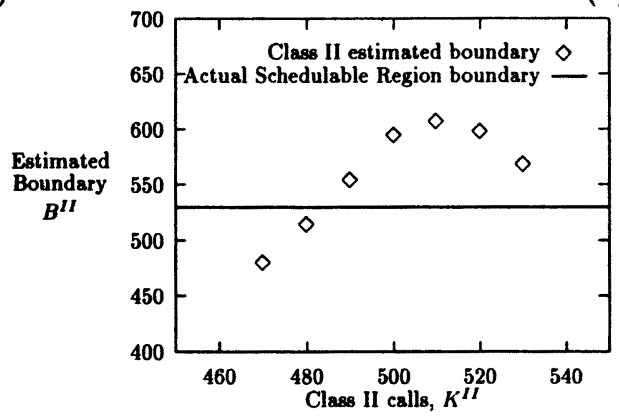

(c)

Figure 8 The schedulable region estimation (SPS scheduler). (a) Video1; (b) Video2; (c) Video3.

Let us turn now to the FIFO scheduler. In this case we not only have to consider Class II delay but also Class I one. In fact, the FIFO discipline does not make any difference between the various traffic classes involved in the multiplexing system, and therefore the schedulable region boundary is found when QoS of any one of them is not guaranteed anymore. Figure 9 shows the results of the estimating mechanism for the two traffic classes (i.e., "॰" for Class I and "+" for Class II). Notice that the lowest one of the two estimations has to be considered as "the" estimation of the boundary of the schedulable region.

\section{CONCLUSIONS}

A framework for a real time estimation of the link capacity in a multiclass network has been presented. A model able to capture delay distribution dy- 


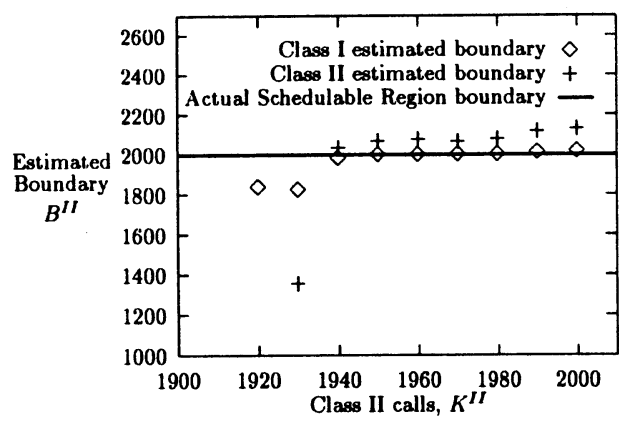

(a)

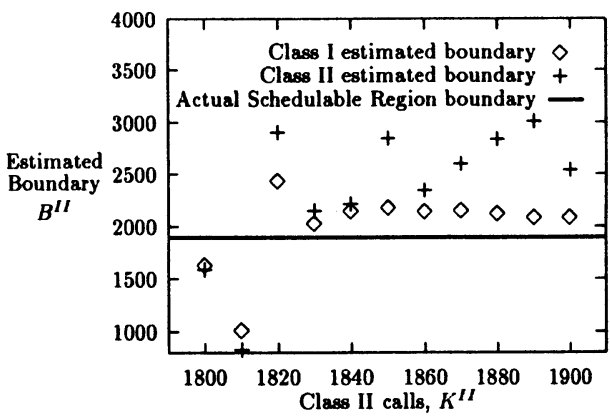

(b)

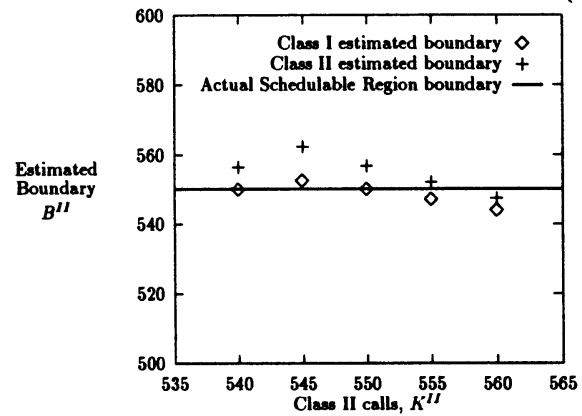

(c)

Figure 9 The model for the delay distribution (FIFO scheduler). (a) Videol; (b) Video2; (c) Video3.

namics has been analyzed and exploited for the estimation task, and numerical results from a number of experiments have been presented.

\section{ACKNOWLEDGEMENT}

The authors would like to thanks Prof. Aurel Lazar who inspired this work and provided thoughtful comments.

\section{REFERENCES}

D. Ferrari and D. C. Verma, "A scheme for real-time channel establishment in wide-area networks," IEEE Journal on Selected Areas in Communications, vol. SAC-8, pp. 368-379, April 1990.

J. M. Ferrandiz and A. A. Lazar, "Admission control of real-time sessions of an integrated node," in Proceedings of the IEEE INFOCOM, pp. 553-559, Bal Harbour, Florida, April 7-11 1991.

R. Guerin, H. Ahmadi, and M. Naghshineh, "Equivalent capacity and its application to bandwidth allocation in high-speed networks," IEEE 
Journal on Selected Areas in Communications, vol. 9, pp. 968-991, September 1991.

J. M. Hyman, A. A. Lazar, and Giovanni Pacifici, "Real-time scheduling with quality of service constraints," IEEE Journal on Selected Areas in Communications, vol. 9, pp. 1052-1063, September 1991.

J.F. Kurose, "On computing per-session performance bounds in highspeed multi-hop computer networks," in Proc. 1992 ACM SIGMETRICS/IFIP Performance'92 Conf., 1992.

J.F. Kurose, "Open issues and challenges in providing quality of service guarantees in high-speed networks," ACM Computer Communication Review, vol. 23, January 1993.

A. A. Lazar, G. Pacifici, and D. E. Pendarakis, "Modeling video sources for real time scheduling," Multimedia Systems, vol. 1, pp. 253-266, April 1994.

A. Parekh and R. Gallager, "A generalized processor sharing approach to flow control in intraged services networks - the single node case," in Proceedings of the IEEE INFOCOM, pp. 915-924, Florence, Italy, May 1992.

\section{BIOGRAPHIES}

Piergiulio Maryni was born in Genoa, Italy in 1965. He received the "laurea" degree in Electronic Engineering and the "Research Doctorate" in Telecommunications from the University of Genoa in 1990, and 1994 respectively. During 1993 he was with the Center for Telecommunications Research (CTR) at Columbia University as a visiting scholar and in 1994 he was with the University of Parma, Italy. He is currently with DIST, University of Genoa. His main research interests are in bandwidth allocation and admission control, both in wired and wireless networks, multimedia communications and services, ATM load estimation.

Giovanni Pacifici received the "Research Doctorate" in Information Science and Telecommunications from the University of Rome "La Sapienza" in 1989. In 1989, he joined the Center for Telecommunications Research at Columbia University were he designed and implemented real-time control and monitoring systems for high speed multimedia networks with quality of service guarantees. In 1995 he joined the IBM T.J. Watson research center, where he his currently involved with multimedia services and service management architectures. Dr. Pacifici is a member of ACM and a senior member of IEEE. 